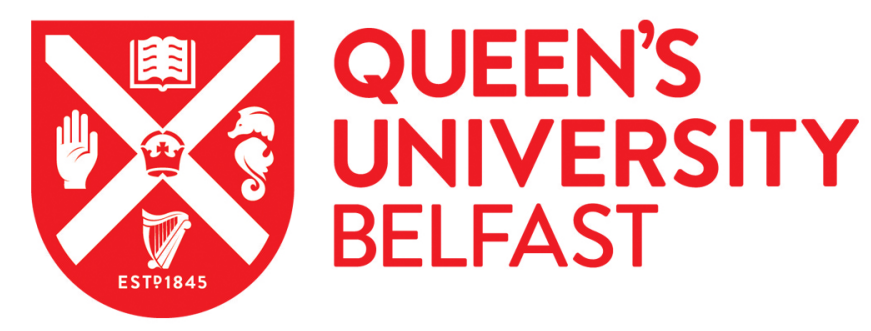

\title{
Accelerating short range MIMO imaging with optimized Fourier processing
}

Fromenteze, T., Yurduseven, O., Berland, F., Decroze, C., Smith, D., \& Yarovoy, A. (2020). Accelerating short range MIMO imaging with optimized Fourier processing. In Passive and Active Millimeter-Wave Imaging XXIII (Vol. 11411). (Proceedings of SPIE). SPIE - The International Society for Optical Engineering. https://doi.org/10.1117/12.2558152

Published in:

Passive and Active Millimeter-Wave Imaging XXIII

Document Version:

Peer reviewed version

Queen's University Belfast - Research Portal:

Link to publication record in Queen's University Belfast Research Portal

Publisher rights

(c) 2020 SPIE.

This work is made available online in accordance with the publisher's policies. Please refer to any applicable terms of use of the publisher.

\section{General rights}

Copyright for the publications made accessible via the Queen's University Belfast Research Portal is retained by the author(s) and / or other copyright owners and it is a condition of accessing these publications that users recognise and abide by the legal requirements associated with these rights.

Take down policy

The Research Portal is Queen's institutional repository that provides access to Queen's research output. Every effort has been made to ensure that content in the Research Portal does not infringe any person's rights, or applicable UK laws. If you discover content in the Research Portal that you believe breaches copyright or violates any law, please contact openaccess@qub.ac.uk. 


\title{
Accelerating short range MIMO imaging with optimized Fourier processing
}

\author{
Thomas Fromentèze ${ }^{\mathrm{a}}$, Okan Yurduseven ${ }^{\mathrm{b}}$, Fabien Berland ${ }^{\mathrm{a}}$, \\ Cyril Decroze ${ }^{\mathrm{a}}$, David R. Smith ${ }^{\mathrm{c}}$, and Alexander G. Yarovoy ${ }^{\mathrm{d}}$ \\ ${ }^{a}$ University of Limoges, XLIM, UMR 7252, F-87000 Limoges, France \\ ${ }^{\mathrm{b}}$ The Centre for Wireless Innovation, The Institute of Electronics, Communications and Information \\ Technology, School of Electronics, Electrical Engineering and Computer Science, Queen's University \\ Belfast, Belfast BT3 9DT, United Kingdom \\ ${ }^{\mathrm{c}}$ Center for Metamaterials and Integrated Plasmonics, Duke University, Durham, North Carolina \\ 27708, USA \\ ${ }^{\mathrm{d}}$ Delft University of Technology, Microwave Sensing, Signals and Systems 2628 CD Delft, The \\ Netherlands
}

\begin{abstract}
In this paper, we describe the recent development of new algorithms applied to short-range radar imaging. Facing the limitations of classical backpropagation algorithms, the use of techniques based on Fast Fourier Transforms has led to substantial image computation accelerations, especially for Multiple-Input Multiple-Output systems. The necessary spatial interpolation and zero-padding steps are still particularly limiting in this context, so it is proposed to replace it by a more efficient matrix technique, showing improvements in memory consumption, image computation speed and reconstruction quality.
\end{abstract}

Keywords: MIMO radar, microwave imaging, millimeter wave imaging, Fourier processing, omega-k algorithm

\section{INTRODUCTION}

Multiple-Input Multiple-Output Imaging (MIMO) is particularly well suited for short-range applications, allowing large synthetic apertures to be formed using a limited combination of transmitters and receivers (Fig. 1).
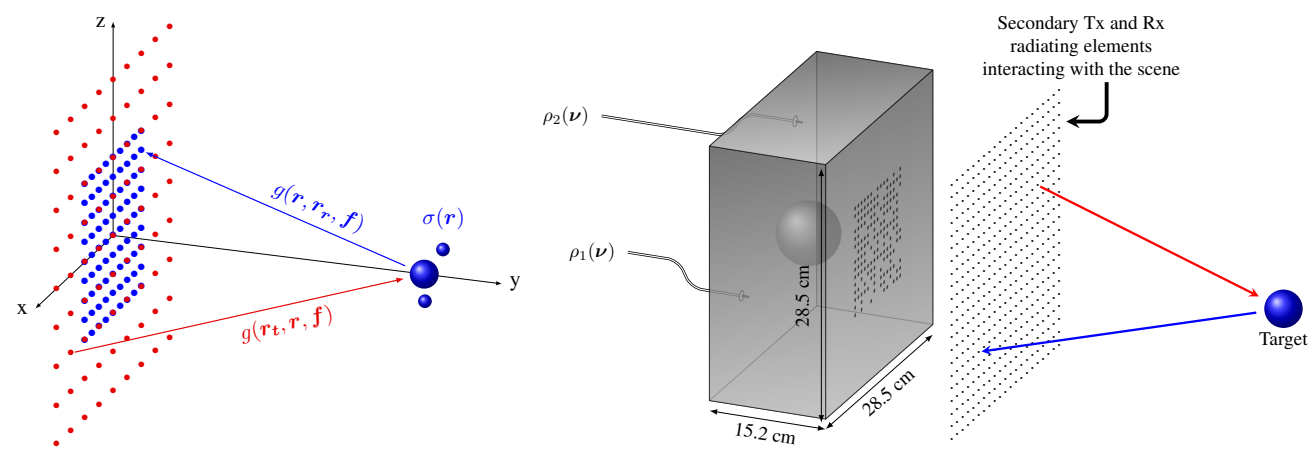

Figure 1: Left: MIMO imaging system, Right: Link between a computational imaging system ${ }^{1}$ with a conventional one. The large number of radiating elements used with these new techniques requires the development of fast image reconstruction algorithms compatible with millions of interactions.

The field of radar imaging is finding an increasing number of applications in terrorism prevention and medical diagnosis. The design of systems with large radiating apertures enables resolutions compatible with these applications, but the need

Further author information: (Send correspondence to Thomas Fromentèze)

E-mail: thomas.fromenteze@ unilim.fr 
for real-time image reconstruction generally precludes the adoption of sequential acquisition strategies. It is therefore necessary to develop radar architectures with a large number of acquisition chains that allow simultaneous measurement of the waves that have interacted with the region of interest. Reconstruction techniques in the Fourier domain such as the Range Migration Algorithm (RMA) have been implemented in this context to accelerate the image reconstruction of synthetic aperture systems ${ }^{2}$ before being adapted to MIMO architectures. ${ }^{3}$ These plane wave expansion techniques were initially developed for sonar imaging applied to Geophysics and proposed by Stolt. ${ }^{4}$ A measurement $s\left(x_{t}, z_{t}, x_{r}, z_{r}, f\right)$ is performed with an array arranged in the plane $y=0$ (Fig. 1) between transmitters of locations $\left(x_{t}, z_{t}\right)$ and receivers of locations $\left(x_{r}, z_{r}\right)$ in order to interrogate a region of interest of reflectivity $\sigma(x, y, z)$. The application of RMA for the reconstruction of this information is obtained by expressing the measurement in the plane wave domain using a Fourier transform on the four spatial dimensions, noted $\mathfrak{F}_{4 D}($.$) , to obtain the following expression: { }^{3}$

$$
\begin{gathered}
\hat{\sigma}_{\text {conv }}(x, y, z) \propto k_{y_{t}} k_{y_{r}} \int_{k_{x}} \int_{k_{y}} \int_{k_{z}} \mathfrak{F}_{4 D}\left(s\left(x_{t}, z_{t}, x_{r}, z_{r}, f\right)\right) \\
e^{j k_{y} y} e^{j k_{x} x} e^{j k_{z} z} d k_{x} d k_{y} d k_{z}
\end{gathered}
$$

with the transmitted and received plane wave components expressed as follows:

$$
\begin{aligned}
& k_{x}=k_{x_{t}}+k_{x_{r}} \\
& k_{y}=\sqrt{k^{2}-k_{x_{t}}^{2}-k_{z_{t}}^{2}}+\sqrt{k^{2}-k_{x_{r}}^{2}-k_{z_{r}}^{2}} \\
& k_{z}=k_{z_{t}}+k_{z_{r}}
\end{aligned}
$$

While this approach allows to reach performances largely exceeding those of the classical Kirchoff migration algorithms, ${ }^{5}$ it remains nevertheless limited by the non-uniform sampling of the longitudinal components of plane waves $k_{y}$ which prohibits the use of fast Fourier transforms in this dimension. It is then possible to use Stolt interpolation techniques, ${ }^{4}$ as well as non-uniform Fourier transforms. ${ }^{6}$ A technique is presented in this paper in order to facilitate these treatments, ${ }^{7}$ allowing substantial gains in memory consumption, computational speed and quality of reconstructed images.

\section{THE TRANSVERSE SPECTRUM DECONVOLUTION TECHNIQUE}

Purely physical considerations can greatly simplify the complexity of treatments associated with MIMO imaging systems. The general principle of the Transverse Spectrum Deconvolution (TSD) technique is based on the fact that a measurement expressed in the plane wave domain is actually the interaction between radiation patterns and the propagated spectrum of the region of interest. For a more intuitive visualization, these patterns are usually presented at each frequency in terms of angular variables $($ theta,$\phi)$ but it is more interesting to work in our context with the transverse plane wave components $\left(k_{x}, k_{z}\right)$. These coordinates make it possible to dispose of radiation patterns by simple spatial Fourier transforms of the field distributions of the radiating apertures used in transmission and reception. The expression is given by the following equation in the case of a grating used in transmission, characterized by a distribution of secondary sources of complex weights $A\left(x_{t}, z_{t}\right)$ (approximated as frequency-independent) :

$$
P_{t}\left(k_{x}-k_{x_{t}}, k_{z}-k_{z_{t}}\right)=\int_{x_{t}} \int_{z_{t}} A\left(x_{t}, z_{t}\right) \exp \left(j\left(\left(k_{x}-k_{x_{t}}\right) x_{t}\right)+\left(k_{z}-k_{z_{t}}\right) z_{t}\right) d x_{t} d z_{t}
$$

Depending on the spatial sampling and the complex magnitude distributions associated with the radiating elements, the radiation patterns exhibit variable directivity and the possible appearance of grating lobes, as shown in Fig. 2. 


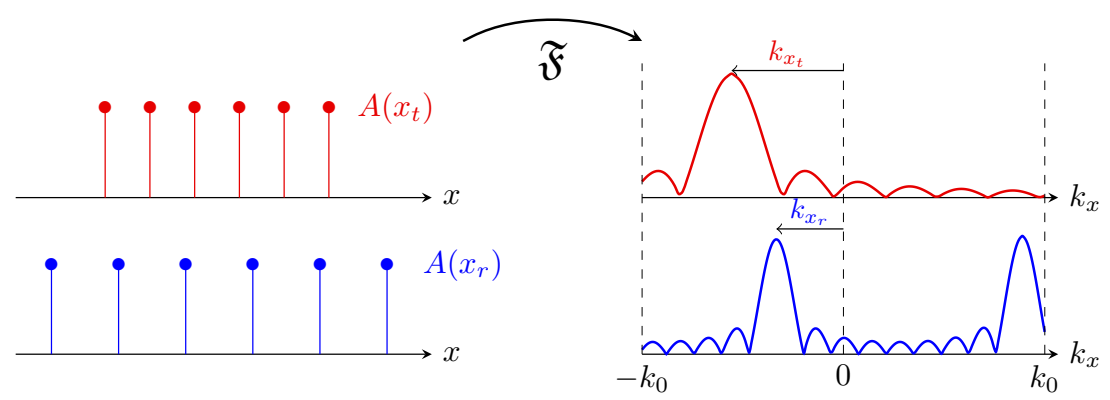

Figure 2: Representation of the relationship between the spatial distributions of the elements forming the radiating apertures and the associated spectra. By applying complex weights to the radiating elements, different directions in space can be interrogated by beamforming, represented here in the Fourier domain.

The general principle based on the measurement of the propagated spectrum of the region of interest using these radiation patterns is described in Fig. 3.

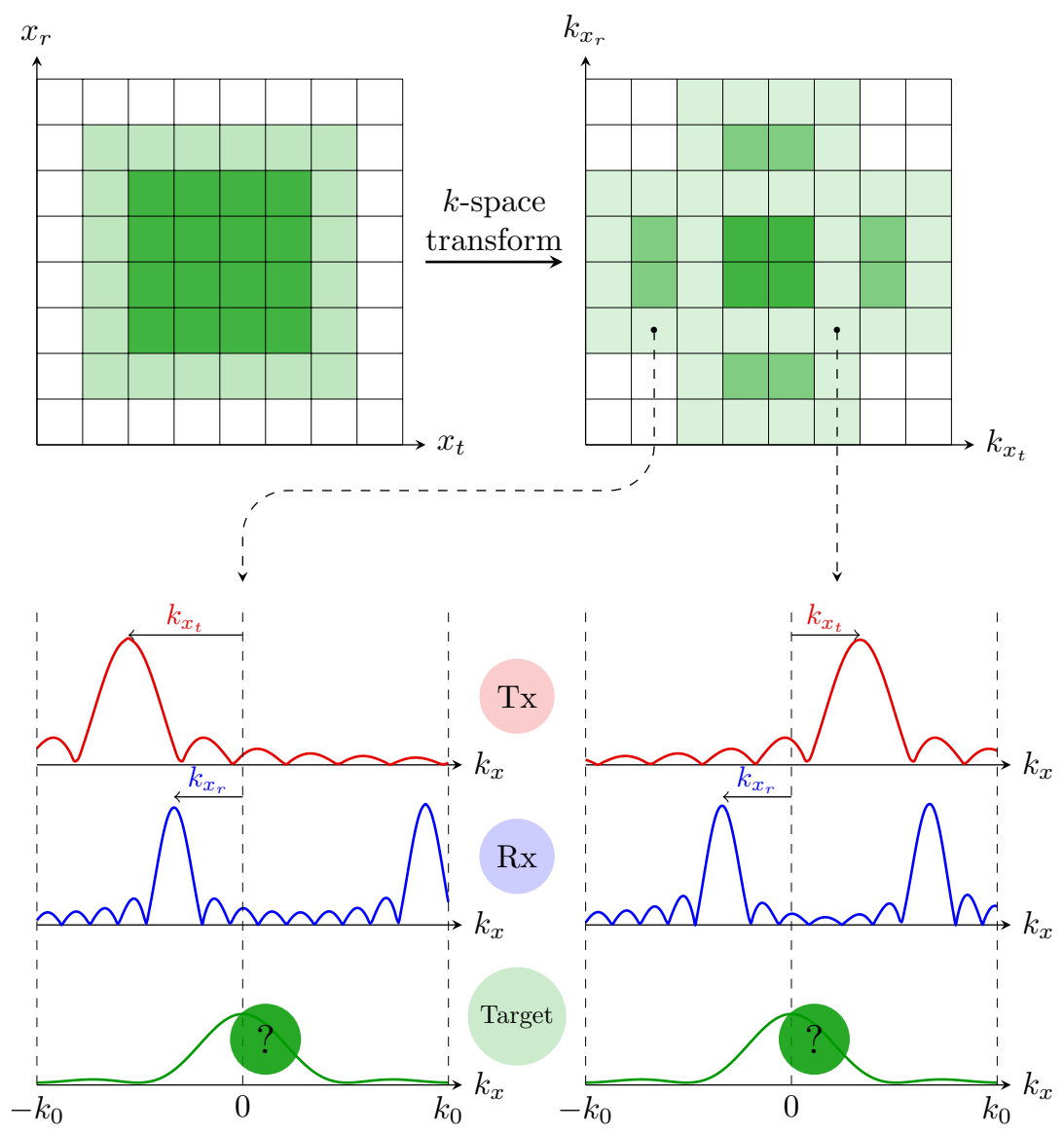

Figure 3: The field distribution measured in space and at each frequency by a MIMO antenna array is expressed in terms of equivalent plane wave components using spatial Fourier transforms. In this domain, each sample corresponds to the interaction between emitted and received radiation patterns with the unknown spectral signature of the region of interest. Translation invariance of antenna patterns expressed in the plane wave domain are exploited here to speed up image reconstructions. 
The measurement expressed with an angular spectrum expansion finally takes the following form :

$$
S\left(k_{x_{t}}, k_{z_{t}}, k_{x_{r}}, k_{z_{r}}, k\right)=\int_{k_{x}} \int_{k_{z}} \underbrace{P_{t}\left(k_{x}-k_{x_{t}}, k_{z}-k_{z_{t}}\right)}_{\text {Tx radiation pattern }} \underbrace{S_{c}\left(k_{x}, k_{z}, k\right)}_{\begin{array}{c}
\text { Propagated spectrum } \\
\text { of the region of interest }
\end{array}} \underbrace{P_{r}\left(k_{x}-k_{x_{r}}, k_{z}-k_{z_{r}}\right)}_{\text {Rx radiation pattern }} d k_{x} d k_{z}
$$

If the position of the radiating elements is with separable variables, it is finally possible to arrange the previous equation as follows :

$$
S\left(k_{x_{t}}, k_{z_{t}}, k_{x_{r}}, k_{z_{r}}, k\right)=\int_{k_{x}} \int_{k_{z}} \underbrace{P_{t, x}\left(k_{x}-k_{x_{t}}\right) P_{r, x}\left(k_{x}-k_{x_{r}}\right)}_{\text {Radiation pattern along } \mathrm{x}} S_{c}\left(k_{x}, k_{z}, k\right) \underbrace{P_{t, z}\left(k_{z}-k_{z_{t}}\right) P_{r, z}\left(k_{z}-k_{z_{r}}\right)}_{\text {Radiation pattern along } \mathrm{z}} d k_{x} d k_{z}
$$

It is then possible to write a matrix formalism to express the link of $S_{c}$ and $S$ for each wavenumber $k$, with the help of the

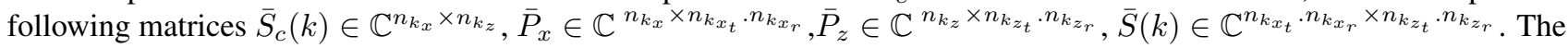
dimensions $k_{x}$ and $k_{z}$ are thus discretized in uniformly sampled vector, defining the maximum extension of the imaging domain. Eq. (7) is then expressed as:

$$
\bar{S}(k)=\bar{P}_{x} \bar{S}_{c}(k) \bar{P}_{z}
$$

The expression of $\hat{\bar{S}}_{c}(k)$ for each value of $k$ thus takes the following form:

$$
\hat{\bar{S}}_{c}(k)=\bar{P}_{x}^{+} \bar{S}(k) \bar{P}_{z}^{+}
$$

where the symbol ${ }^{+}$stands for the pseudo-inverse operator.

Having expressed all the transverse components of plane waves along the same dimensions $k_{x}$ and $k_{z}$, it is finally possible to determine the expression of the longitudinal components such as ${ }^{7}$ :

$$
k_{y}=2 \sqrt{k^{2}-k_{x}^{2}-k_{z}^{2}}
$$

The reflectivity function of the target can finally be estimated :

$$
\hat{\sigma}_{\text {tsd }}(x, y, z) \propto \int_{k_{x}} \int_{k_{z}} \int_{k} k_{y}^{2} \hat{S}_{c}\left(k_{x}, k_{z}, k\right) e^{j\left(k_{x} x+k_{z} z+k_{y} y\right)} d k d k_{x} d k_{z}
$$

Thanks to this approach, the merging of transverse dimensions is greatly simplified by physical considerations, allowing to perform the backpropagation operation by phase compensation of plane waves on a $\hat{\bar{S}_{c}}(k)$ matrix having only 3 dimensions instead of 5. Compared to the initial implementation of MIMO RMA, ${ }^{3}$ the algorithmic complexity is greatly reduced, while allowing to take into account all the plane wave components in order to improve the reconstructions. An analysis of the performance of this technique is proposed in the following section.

\section{RESULTS}

A first validation is proposed to illustrate the numerical performance of the proposed approach. An experiment is simulated in the $\mathrm{K}$ band $(18-26 \mathrm{GHz})$ sampled by $n_{f}=20$ frequency points, using a radiating aperture composed of $n_{b a}^{2}$ radiating elements used in both transmission and reception, i.e. $n_{b a}^{4}$ interactions at each frequency. These antennas are spaced 0.8 $c / f_{\max }=9.2 \mathrm{~mm}$ apart according to the transverse dimensions $x$ and $z$. The calculations are carried out with Matlab, using a computer equipped with $16 \mathrm{~GB}$ of RAM and a 6-core $3.5 \mathrm{GHz} \mathrm{CPU}$. The methods considered and the computation times obtained by their application are given in Fig. 4 and the imaging scenario is shown in Fig. 5. These simulations are studied in the particularly unfavourable case of dense antenna arrays in order to correspond to the conditions associated with the use of computational techniques, ${ }^{1,8}$ made compatible with this digital processing by the use of equalization techniques adapted to MIMO architectures. ${ }^{9}$

This first study highlights a significant gain in computing time by applying the new plane wave fusion technique, which increases with the size of the problems studied. The application of TSD RMA is optimized by coupling it with Stolt 

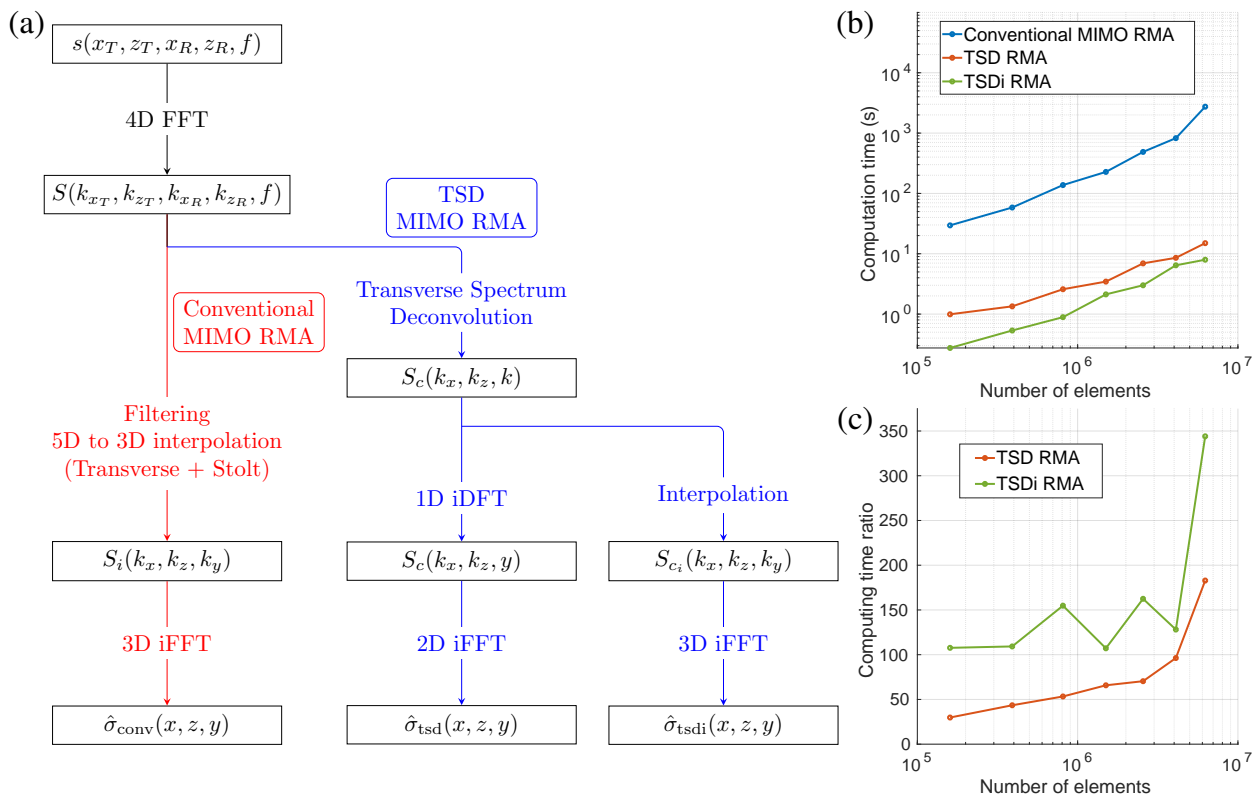

Figure 4: (a) Illustration of the studied algorithms: the conventional RMA ${ }^{3}$ is modified by applying the proposed TSD technique. It is then possible to reconstruct the depth by Direct Fourier Transform or by Stolt interpolation. (b) Computation times obtained for different radiating apertures composed of $n_{b a}=10^{2}$ to $40^{2}$ radiating elements, leading to $n_{f} \cdot n_{b a}^{4}$ measured interactions. (c) Ratio of computation times obtained, taking the conventional RMA as a reference.

interpolation, allowing to reduce the processing associated with the backpropagation step initially computed by discrete Fourier transform of the non-uniform plane wave component along the $y$ axis (Eq. (11)).The application of this technique is illustrated in comparison with the classical MIMO RMA, ${ }^{3}$ attesting to the good performance of the proposed approach without any need for data preconditioning according to the antenna dimensions by interpolation and zero-padding (Fig. 5).
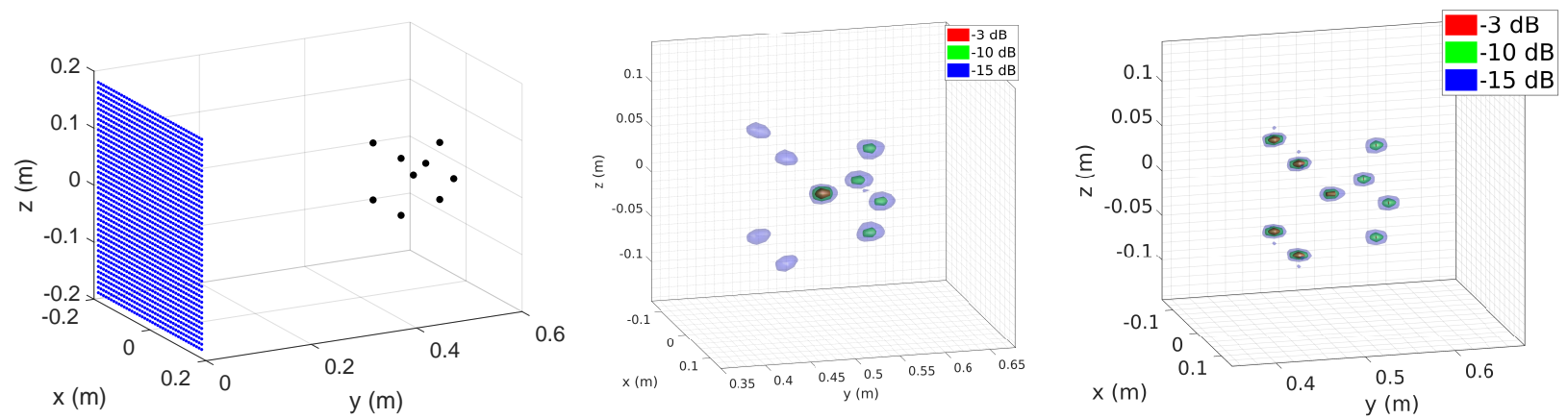

Figure 5: Left: An array of $40^{2}$ uniformly spaced radiating elements is used for both transmit and receive. 9 target points are arranged at a distance of $0.5 \mathrm{~m}$ on an extension of $10^{t} \mathrm{extcm}^{3}$. Center: Reconstruction by application of conventional RMA. ${ }^{3}$ Right: Reconstruction by the TSD RMA associated with a Stolt interpolation. Matrix fusion of the transverse components of plane waves allows better image reconstruction without the need for computationally intensive interpolation and zero-padding techniques.

Finally, RMA TSD is applied to a practical case, imaging a more complex scene representing a man carrying a firearm in a context of detection of hidden objects under clothing considered transparent at these frequencies (Fig. 6). The simulations are performed using an array of $71^{2}$ radiating elements used in transmission and reception in the $18-26 \mathrm{GHz}$ band. The antennas are spaced at $0.7 \lambda_{\min }=6.92 \mathrm{~mm}$. The target is placed at $1 \mathrm{~m}$ and consists of 67455 facets with a reflectivity of 0.8 , chosen sufficiently small in relation to the dimensions of the radiating aperture to allow an physical optics approximation. 


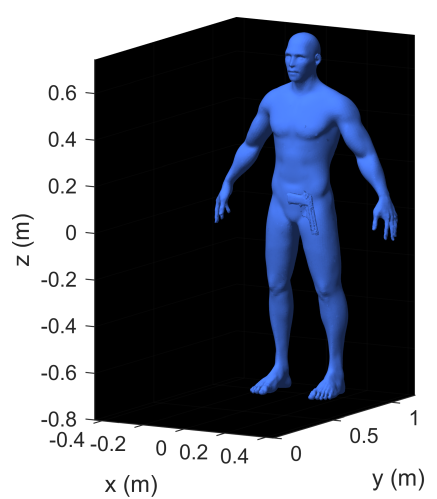

(a)

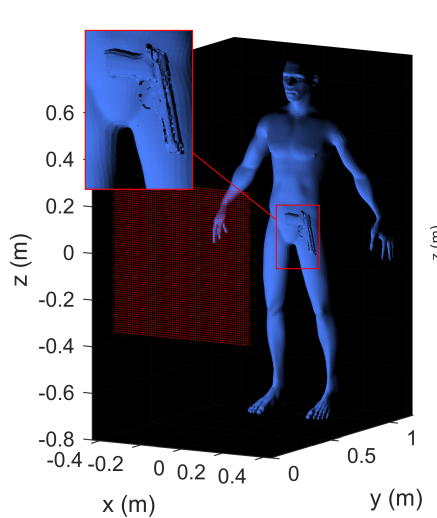

(b)

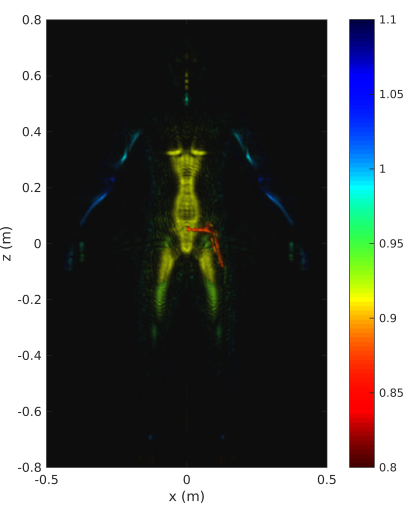

(c)

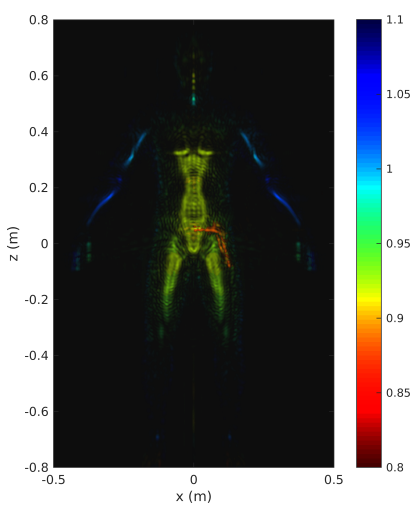

(d)

Figure 6: (a) Target imported into a Matlab radar simulation engine. (b) Calculation of the interactions between the facets and the radiant aperture at each frequency. The results obtained by applying TSD RMA (c) and TSDi RMA (d) are represented by color coding the depth.

The reconstructions are performed in $27.1 \mathrm{~s}$ by TSD RMA and $24.8 \mathrm{~s}$ by TSDi RMA on the computer presented above. Note that in each case, $15 \mathrm{~s}$ are necessary for the only FFT steps that could be accelerated on highly parallelizable material. The impact of Stolt interpolation is visible on the reconstruction but still allows a clear reconstruction of the image and identification of the position of the firearm.

\section{CONCLUSION}

A new short range MIMO imaging technique has been described in this paper following a first publication. ${ }^{7}$ The results allow us to anticipate applications in many contexts requiring large volumes of data to be processed rapidly. Partitioning strategies may allow to further accelerate reconstructions ${ }^{10}$ and future efforts will enable these algorithms to be ported to more efficient and highly parallelizable architectures in order to ensure their real time application.

\section{REFERENCES}

[1] Fromenteze, T., Yurduseven, O., Imani, M. F., Gollub, J., Decroze, C., Carsenat, D., and Smith, D. R., "Computational imaging using a mode-mixing cavity at microwave frequencies," Applied Physics Letters 106(19), 194104 (2015).

[2] Lopez-Sanchez, J. M. and Fortuny-Guasch, J., "3-d radar imaging using range migration techniques," IEEE Transactions on antennas and propagation $\mathbf{4 8}(5), 728-737$ (2000).

[3] Zhuge, X. and Yarovoy, A. G., "Three-dimensional near-field mimo array imaging using range migration techniques," Image Processing, IEEE Transactions on 21(6), 3026-3033 (2012).

[4] Stolt, R. H., "Migration by fourier transform," Geophysics 43(1), 23-48 (1978).

[5] Maijala, P. et al., "Application of some seismic data processing methods to ground penetrating radar data," in [Fourth International Conference on Ground Penetrating Radar], cp-303, European Association of Geoscientists \& Engineers (1992).

[6] Wang, J., Aubry, P., and Yarovoy, A., "3-d short-range imaging with irregular mimo arrays using nufft-based range migration algorithm," IEEE Transactions on Geoscience and Remote Sensing (2020).

[7] Fromenteze, T., Yurduseven, O., Berland, F., Decroze, C., Smith, D. R., and Yarovoy, A. G., "A transverse spectrum deconvolution technique for mimo short-range fourier imaging," IEEE Transactions on Geoscience and Remote Sensing 57(9), 6311-6324 (2019).

[8] Fromenteze, T., Yurduseven, O., Boyarsky, M., Gollub, J., Marks, D. L., and Smith, D. R., "Computational polarimetric microwave imaging," Optics express 25(22), 27488-27505 (2017).

[9] Fromenteze, T., Kpré, E. L., Carsenat, D., Decroze, C., and Sakamoto, T., "Single-shot compressive multiple-inputs multiple-outputs radar imaging using a two-port passive device," IEEE Access 4, 1050-1060 (2016).

[10] Álvarez, Y., Rodriguez-Vaqueiro, Y., Gonzalez-Valdes, B., Las-Heras, F., and García-Pino, A., "Fourier-based imaging for subsampled multistatic arrays," IEEE Transactions on Antennas and Propagation 64(6), 2557-2562 (2016). 